\title{
“走出去”的地理学 一从人文地理学视角看中非合作研究
}

\author{
安 宁 ${ }^{1}$, 梁邦兴 ${ }^{2}$, 朱 竑 ${ }^{1 *}$ \\ (1. 广州大学 地理科学学院 华南人文地理与城市发展研究中心, 广州 510006 ; \\ 2. 华南师范大学地理科学学院,广州 510631)
}

\begin{abstract}
摘 要: 中非合作正在成为国际政治和经济舞台上的重要事件。本文选用Endnote 和Antconc等信息提取软件对国 内外知名文献库中收录的有关中非合作的文章进行了梳理。以“中非合作”作为模糊关键词, 对 Web of Science、 Taylor \& Francis、Elsevier 和中国知网四大数据库进行搜索, 得到相关中外文文献共计 623 篇。分析发现, 中外学者 对中非合作议题的关注多集中于政治学和经济学的学科分析框架之下, 其他学科视角相对缺乏。从人文地理学视 角来看,尽管也有部分经济地理和政治地理学者对该话题进行了探讨,但并未完全脱离政治学和经济学的分析框 架, 且数量较少、未能体系化。因此, 本文从与中非合作最为紧密的政治地理、旅游地理以及社会文化地理等三个 方向提出了未来可能成为中非合作研究热点话题的基本研究框架。鉴此, 随着国家“一带一路”倡议的逐步实施, 期望未来能有更多人文地理学者投入到中非合作研究中, 积极发挥中国地理学 “走出国门”的大国智力责任和担 当,为中非合作研究与实践作出自己的贡献。
\end{abstract}

关 键 词: 中非合作; 人文地理; 文献分析; 走出去

\section{1 引言}

在地理学界有一个现象, 发达国家地理学者的 研究范围并没有很强的地域局限性, 研究议题往往 涉及全球各个角落。而中国地理学研究在一段时 间里却主要局限在本国范围内。

随着中国共建“一带一路”倡议的提出和实施, 中国地理学者开始比以往更为关注中国以外的地 理学研究话题, 如杜德斌等(2015)从地缘政治学的 角度分析了 “一带一路” 倡议在南海、中亚以及欧亚 大陆桥地带等地区面临的可能风险; 孟辽阔(2015) 讨论了巴基斯坦作为中国传统友好国家和“一带一 路”沿线重要国家的双重角色, 提出了将巴基斯坦 打造成为具有 “中国方案精神” 新型国际关系示范 点的建议; 陆瑾(2015)采用话语分析的方法, 从地缘
政治、经济和文化等角度研究了伊朗人对“一带一 路” 的认知等。上述研究一定程度上可视为中国地 理学者及其研究“走出去”的尝试。然而, 随着中国 综合国力的不断增强, 以及为满足日渐深人的参与 全球治理的需求, 亟需中国学者在智库保证层面给 予更多的支持。而地理学无论是为国家建设服务 之用, 还是满足学科自身发展之需求, 走出国门开 展全球视野下的研究都显得尤为必要。

中非交往历史由来以久。自 1956年中国与埃 及正式建交以来, 中非关系不断发展, 之后因第三 世界间的友谊开始快速升温, 双方合作不断深化。 直至 1980 年, 中非合作项目超过了 800 项(Manji et al, 2007)。自 2000 年中非合作论坛举办以来, 中非 关系更是进人了新的历史阶段: 从 2007 年开始, 中 国就超越法国,成为非洲进口产品的首位输出国;

收稿日期:2018-04-11; 修订日期:2018-11-14。

基金项目: 国家自然科学基金项目(41630635,41701149,41871127) [Foundation: National Natural Science Foundation of China, No.41630635, No.41701149, No.41871127]。

作者简介:安宁(1987-), 男, 陕西汉中人,博士,主要从事政治地理研究,E-mail: ning_ann@126.com。

通讯作者:朱竑(1968-),男,甘肃临夏人,教授,主要研究领域为社会文化地理,E-mail: zhuh@scnu.edu.cn。

引用格式: 安宁, 梁邦兴, 朱竑. 2018. "走出去"的地理学: 从人文地理学视角看中非合作研究[J]. 地理科学进展, 37(11): 1521-1532. [An N, Liang B X, Zhu H. 2018. Chinese geography "goes out": Looking at China-Africa cooperation from the perspective of human geography [J]. Progress in Geography, 37(11): 1521-1532.]. DOI: 10.18306/dlkxjz.2018.11.008 
截至 2016 年, 中国在非修建铁路里程达 $5675 \mathrm{~km}$, 公路 $5064 \mathrm{~km}$; 截至 2018 年 10 月, 中国已与 51 个非 洲国家全面建交; 从政治、经济、文化、教育和科技 等多个方面, 中国都在全面深化对非合作关系(央 视网, 2016)。此外, 自中国 “一带一路”倡议提出以 来, 非洲成为不可或缺的参与者之一, 中非合作将 进一步加快(王南, 2015)。中非合作正成为全世界 无法忽视的重要地理、经济和政治事件, 催生了许 多与之相关且值得广泛关注的研究议题。

从另一个角度来说, 迅猛发展的中非合作正在 触动西方国家的敏感神经。西方政府、媒体、学界 等纷纷发出声音, 质疑中非合作, 不惜用“中国威胁 论” 以及 “新殖民主义”等词藻对中非合作进行抨击 (王洪一，2006; Mawdsley, 2008; 罗建波, 2009; Jauch, 2011)。虽然中国政府和媒体都在强调中非 合作是 “南南合作”的经典样板以及互利共赢的新 型国际关系模式的有力见证, 但整体来说缺乏像西 方一样的强有力的话语权以及缺乏系统的学理支 撑。西方一手炮制的 “中国威胁论” 给中国在国际 舞台的形象带来不少负面影响, 甚至为中国在非洲 的政治经济活动带来了困扰和阻力。为此, 需要中 国学者加强对此类问题的探讨, 积极发出关于中非 合作的声音, 针对西方对中非合作污名化的现象做 出必要的回应。

上述分析说明, 中非合作研究既是对快速发展 的中非关系智力层面支持的强烈需求, 又是回应西 方对中非合作污名化的必然工作, 同时也是中国地 理学研究走出国门的契机之一。基于此, 本文通过 国内外知名文献检索库(Web of Science、Taylor \& Francis、Elsevier 和中国知网)关于中非合作的文献 梳理和分析, 对已有关于中非合作的研究进行评 述, 提出从人文地理学视角研究中非合作可能涉及 的议题, 以期中国学者在未来关于中非合作的研究 方面发出更加响亮的“中国声音”。

\section{2 数据和方法}

本文采用了中外文核心文献检索库分析的方 法探讨中非合作的研究现状。对于外文核心文献 检索库, 研究将 Web of Science(其中涵盖Wiley, Springerlink, Sage 和 Routledge 等数据库), Taylor \& Francis 以及 Elsevier 作为观测对象, 以 “中非合作” （包括 “Sino-Africa cooperation”, “China- Africa cooperation”, "Sino-Africa collaboration” 和 “China-Af- rica collaboration”等四组搭配词)作为关键词进行 主题、关键词和摘要关键词检索; 而对于中文核心 文献检索库, 研究将中国知网中文核心库作为文献 数据源, 以“中非合作”作为关键词对数据库进行主 题、关键词和摘要搜索(中外文文献搜索时间段都 是截至 2017 年 12 月 31 日)。为了弥补可能的数据 缺漏, 在搜集过程中, 以部分相关高引论文作为补充。

基于上述方法, 本文获得与中非合作相关的外 文文献 241 篇、中文文献 382 篇。将搜集到的文献 导人 Endnote 进行分析后发现: 从中非合作研究的 出版物情况来看, 外文文献期刊来源较为分散, 共 有 127 种期刊发表了中非合作研究成果, 其中 《South African Journal of International Affairs》最多, 达 12 篇; 中文文献期刊来源相对较为集中, 有 71 种 期刊发表了相关研究论文,但主要集中在《西亚非 洲》和《国际政治研究》,两种期刊共发表了 191篇中 非合作文章; 从学者的学术影响力来说, 刘鸿武、李 安山、罗建波、黄梅波和张春等中国学者学术标签 较为明显, 均发表了超过 10 篇相关文章, 但大部分 是非地理学者; 从外国学者来说, 都柏林三一大学 的 Padraig 学术标签较为明显, 发表了 6 篇相关文 章, 而且是基于地理学的学科背景展开的。从中非 合作研究的历史脉络来说, 中文期刊对该话题的关 注较早,始于 20 世纪 90 年代初,而外文期刊的真正 关注则始于 21 世纪初。在 2008 年前后, 中外期刊 对中非合作研究的关注度开始迅猛增加(图 1)。此 外, 通过Antconc 软件对文献标题进行分析可得, 中 文期刊都围绕“中非关系”、“发展”、“投资”、“援助” 和“中非经贸合作”等主题展开, 而外文文献则主要 围绕“Economic”(经济)、“China-Africa relation”(中 非关系)、“Aid”(援助)、“Agriculture”(农业)和“Me-

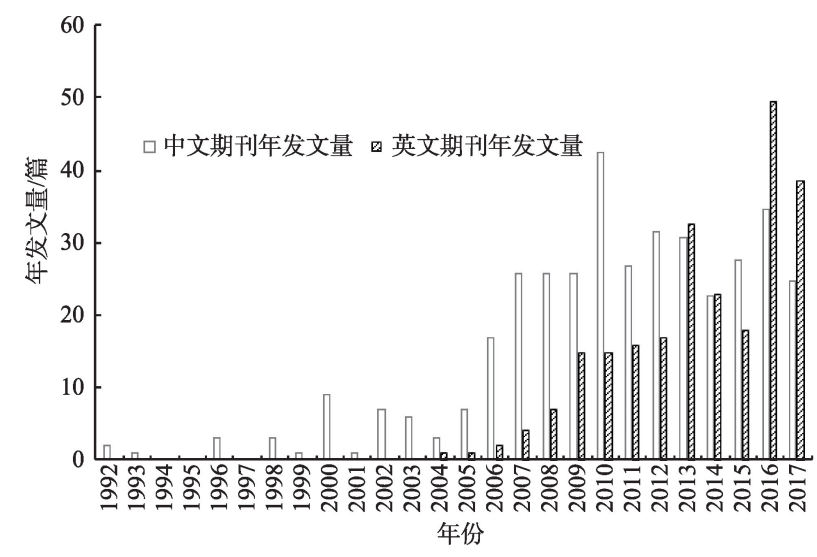

图 1 中非合作年度文献量统计图

Fig.1 Annual number of China-Africa cooperation literature 
dia” (媒体)等主题展开(表 1)。由此可以得到一个比 较初步的结论, 即中外学者对中非合作主要的关注 点是围绕政治和经济研究开展的。

\section{3 中非合作研究评述}

\section{1 中、西、非学者对中非合作议题的关注}

(1) 中国学者对中非合作议题的关注

国内关于中非合作的研究起步较早, 现有知网 数据显示可追溯到 1992 年黄泽全(1992a)在《西亚 非洲》上发表的《中非经济合作与文化交流》一文。 但 21 世纪之前, 受限于中非合作尚未全面展开的现 实情况以及科学研究实用性的导向等原因, 中非合 作相关的研究成果数量比较少, 主要是少数学者对 中非合作历程宏观性的回顾和总结(黄泽全, 1992a, 1992b; 傅政罗, 1996; 汪勤梅, 1998)。随着 2000 年 中非合作论坛的成功举办, 中非合作进入常态化阶 段。中国学者对中非合作的研究开始快速增加。 从搜集获取的文献标题词来看, 国内学者主要围绕 “中非经贸合作” “投资” 和 “援助” 等主题展开分 析。从学者的学科背景以及研究的分析框架来看, 国内学者主要从经济学、政治学以及教育、文化和 语言等学科角度进行讨论。

首先, 从经济学研究角度出发, 国内学者们更 多考虑的是经济合作、投资和援助的模式架构, 以 及根据不同国际经济状况对中非经济合作、投资和 对非援助带来的经济影响分析等。如姚桂梅 (2008, 2013)分析了中国对非洲投资合作的主要模
式及挑战, 并讨论了中非合作论坛在推动中非经贸 合作的跨越性发展过程中的作用和意义; 张菲 (2013)探讨了作为中非经贸合作重要载体的中非经 贸合作园区的建设模式以及当前存在问题和解决 建议; 汪文卿等(2014)利用索洛增长模型分析,得出 中国的投资和援助对非洲经济增长产生着正向显 著影响的结论。中国学者还针对中非合作中的经 济行业进行了研究, 如张忠祥(2009)讨论了中非合 作论坛框架下的农业合作模式, 指出中非农业合作 很大程度促进了非洲国家社会经济的发展和进步; 贺鉴等(2017)探讨了中非如何就非洲丰富的天然渔 业资源进行合作, 以及合作中的机遇与挑战; 黄梅 波等(2016)分析了中非产能合作的金融需求以及中 非金融合作的推进模式,包括人民币国际化在非洲 遇到的困境等。

其次, 从政治学研究角度出发对中非合作相关 主题进行讨论也是中国学者研究的重点。整体来 说,这类研究倾向于将中非合作置于 “南南合作”的 政治框架下进行讨论。南南合作主要是广大发展 中国家(由于大多数都位于地球南半球或北半球的 南部, 故而称为 “南南合作”)基于共同的历史遭遇 而开展的,相互之间具有平等地位, 旨在相互分享 和共同进步的技术和经济领域的交流合作框架(黄 梅波等, 2013)。从这一角度出发,张海冰(2006)总 结了中国对非洲援助的特点,包括不带政治附加条 件、优先考虑非洲国家的实际需要和促进非洲经济 发展等,因此认为中非合作主要是基于 “南南合作” 的框架实施的; 罗建波(2013)阐述了中国对非洲的

表 $1^{(1)}$ 中非合作研究标题高频词及词频统计(前 10)

Tab.1 High frequency words and statistics of the titles of China-Africa cooperation literature (top 10)

\begin{tabular}{clllc}
\hline 序号 & 中文高频词 & 词频 & 外文高频词 & 词频 \\
\hline 1 & 中非关系 & 50 & Economic & 26 \\
2 & 发展 & 42 & Aid & 13 \\
3 & 投资 & 37 & China-Africa relation & 11 \\
4 & 援助 & 37 & Agriculture & 9 \\
5 & 研究 & 37 & Media & 6 \\
6 & 中非经贸合作 & 26 & Migrants & 6 \\
7 & 现状 & 24 & South-South cooperation & 6 \\
8 & 分析 & 21 & Diplomacy & 6 \\
9 & 影响 & 18 & Inverstment & 6 \\
\hline
\end{tabular}

(1)注: 为保证所得高频词的有效性,作者排除了中国、非洲、中非合作、China、Africa、China-Africa cooperation、Sino-Africa cooperation、ChinaAfrica collaboration 和 Sino-Africa collaboration 等词的统计,并将外文意思相同词性变化的词进行合并统计。 
外交本质, 指出中国在非洲有自身的战略诉求, 这 一点冊庸讳言, 但是中非关系一开始就是以南南合 作和亚非复兴为指向的; 刘鸿武团队阐明了中国援 非五十年是南南合作理念的重要实践, 并表明近年 来中非命运共同体概念的建构是对中非合作和南 南合作表述的发展(胡美等, 2012; 刘鸿武等, 2013)。

除经济和政治的分析视角以外,近年来随着中 非合作的日渐深人, 中国学者对中非合作相关话题 的研究视角也不断扩展, 逐渐开始涉及从教育学、 语言学、文化研究和 “一带一路”倡议等角度对中非 合作议题展开讨论。例如, 牛长松(2014)以在非的 孔子学院作为案例, 讨论了建立语言文化外交的综 合路径, 发现汉语语言和文化教学提升了非洲人对 中国正面和积极的国家形象, 提高了中国在非影响 力, 增进了中非之间的交流(并不局限于语言和文 化); 李安山(2015)则研究了中非民间交往的历史演 变过程及其特征, 指出中非民间交流体现了文化馈 赠、文化互鉴和文化融合的思想, 正是由于民间双 向友好交往, 理解对方文化, 才为中非合作的广泛 开展打下坚实的基础; 在 “一带一路”倡议提出的大 背景下, 周文等(2017)借助对比多个经济体(欧盟、 中国、美国、印度和日本等)与非洲国家间的贸易和 投资数据, 发现 “一带一路”倡议下的中非合作对非 洲而言是合作共赢、共同发展的关系, 并非西方中 心论建构的新殖民主义实践。

(2) 西方学者对中非合作议题的关注

相对于中国学者来说, 西方学者对中非合作议 题的关注起步较晚。具体而言, 自 2000 年以来, 随 着中非合作论坛的举办及中非合作在国际上影响 力的逐步增强, 相关话题才开始吸引大量海外学者 的关注, 才开始真正投人中非合作研究。从西方学 者对中非合作议题讨论的相关标题分析来看, 西方 学者主要围绕“Economic”(经济)“China-Africa relation” (中非关系) “Aid” (援助) “Agriculture” (农业) “Media”(媒体) 和 “Migrants” (移民)等主题而展开 的。从学者的学术背景以及研究的分析框架来看, 西方学者对中非合作的探讨与中国学者的分析框 架大体一致, 都偏好从经济或政治的分析视角进行 讨论。

首先, 与中国学者相似的是, 西方学者也习惯 将中非合作置于经济研究框架中进行分析, 探讨商 品、经济援助、农业、移民等经济行为及其产生的影 响, 如经济增长、社会效应和环境影响等。例如,
Jaff等(2002)认为石油资源是维系中非合作的重要 支撑点, 非洲丰富的自然资源可为中国提供快速发 展必需的能源; Montinari 等(2011)讨论了中国在非 洲的经济增长及其对撒哈拉以南非洲农产品的出 口的影响,指出中国在非洲的经济增长对其他国家 粮食价格及非粮食出口产生了一定的负面影响; Kärkkäinen(2016)通过分析三家中资企业(中国北方 工业公司、安徽省外经建设(集团)有限公司、中国机 械设备工程股份有限公司)于 2000-2013 年期间在 津巴布韦自然资源方向的投资情况,讨论了投资遭 受阻碍因素及其对当地经济的负面经济影响; Lee (2009)通过对中国移民与非洲当地居民之间冲突所 产生的飞地进行分析, 从管理的角度指出, 伴随中 国经济增长而来的人口迁徙给非洲政府管理带来 了极大的挑战; Berger(2006)更是直接指出中国从 非洲日益增加进口能源资源损害了西方国家的利 益; Haglund(2009)讨论了中国在赞比亚的铜矿企业 对赞比亚环境的影响,籍此讨论了企业的社会责 任等。

此外,西方学者对中非合作的政治经济影响也 颇为关注。但是, 与中国学者所强调的“南南合作” 分析框架极为不同的是, 有相当一部分西方学者认 为,中非合作更多的是基于中国自身的经济和政治 利益诉求而非平等共享的技术和经济分享模式。 例如, Naim (2007)极端地认为, 中国对非援助形式 和内容均与以经合组织为代表的国际援助有着很 大的区别, 中国对非援助是在不透明的情况下进行 的, 更为重要的是其最终目的并非为推动非洲国家 真正的进步,而是关注自身的获利与否, 因而将中 国对非的援助丑化为 “流讯援助”; 类似地, Mung (2008)认为现今中国对非政策主要是立足于获取自 然资源、扩大出口市场以及增加外交支持等目的。 Campbell (2008)引援了 Mung 的观点, 认为中国在 非洲的经济行为是不负责任的“殖民主义”行为, 其 最终只有纯粹的政治和经济目的, 即为获取石油和 战略矿产、扩大市场、培育合作伙伴甚至是获取未 来的军事支持, 以及挑战西方国家在非洲的地位, 尤其是美国的全球霸主地位做准备。与上述观点 相比, Tan-Mullins 等(2010)的观点则相对缓和务实, 他们认为中国在非洲的经济行为并非殖民主义实 践, 而是全球化背景下的一种“新资本主义”, 中国 并非掠夺资源和政治资本的殖民者,但也不是建构 “南南合作”的平等贸易, 似乎更加倾向于一种资本 
的输出和对海外市场的占领。在大部分西方学者 眼里, 中国不干涉他国内政的外交政策也正在含糊 地变化, 中非合作权力的不平衡也日趋明显(Larkin, 1973)。鉴于西方社会对华敌意的刻板共识, 在 研究中更多基于经济剥削、政治地位不平等角度出 发进行剖析, 所以容易产生上述零和博弯式的结 论。因西方学者长期占据着国际学术话语权, 其言 论快速向全世界扩散, 这也是“新殖民主义” “流讯 援助” “新资本主义”等错误言论在国际上有相当一 部分学术市场的原因。

当然, 负面评价并非西方学者的全部学术认 知, 也有部分西方学者持有相反或相对中立的观 点。例如, Meidan (2006)通过中国对非政策解读, 得出相比于其他国家更为强烈的政治意图, 中国对 非洲现行的政策主要以“商业为主, 政治暂时搁置” 的观点。此外, 随着中非合作项目的增加及其细节 日渐透明, 少数西方学者开始认为 “中非合作” 是一 个有益的尝试。例如, Sautman 等(2007)讨论了中国 对非洲的农业援助, 提出中非合作既是一种朋友关 系也是一种利益关系的观点:一方面中国的确给非 洲带来了与西方国家不同的机遇, 另一方面中国对 非洲的援助、移民、投资和基建等也是基于其特定 的政治经济诉求。此外, Carmody等(2007)认为, 现 今中美在非洲的地缘经济竞争有助于改造非洲原 来的殖民地贸易结构, 尽管这有可能引发中、美在 非洲的冲突; Asche(2008)认为虽然中国进人非洲的 真正的受益者不能确定, 但是中国不同于西方国家 的合作模式(非洲模式)对非洲而言是一个不错的尝 试, 西方政府学者不应对中国的影响一味地进行批 判; Grimm 等(2017)运用三个非洲国家的例子, 说明 中非合作不仅没有削弱欧盟在非洲的集体利益, 反 而促进了其发展, 欧盟应当将中国在非洲投资活动 作为检验自身及其倡导多边共同发展策略的案例等。

(3) 非洲学者对中非合作议题的关注

相比中国和西方学者对中非合作议题的关注, 本文发现还有一个不可忽视的群体: 作为中非合作 实践的重要参与者, 非洲学者群体对相关议题的关 注也是比较密切的。然而, 因其所处的特殊背景, 非洲学者对中非合作话题的关注点有所不同, 其声 音更为多元、复杂, 甚至有相互矛盾的现象。部分 学者的观点或与中国学者的主流话语相呼应, 部分 受西方学者的观点影响, 抑或是从非洲的发展出发 提出独立于中西学者之外的独特观点。
具体来说,有一部分非洲学者支持中国主流话 语所构建的中非 “南南合作” 的观点。例如,博茨瓦 纳学者Osci-Hwede(2012)认为中国正在非洲进行与 西方不同的尝试和探索,试图消除中非之间不对称 的经贸关系,建立一种合作而非剥削的关系; 此外, 喀麦隆学者Amadi (2012)则认为, 非洲现在和冷战 时期一样需要明确自身的地缘政治定位,拥护北京 的“不干预政治”的态度，但是对“南南合作”则持怀 疑态度, 建议非洲摆脱政治援助来发展, 同时建议 中国应塑造负责任的大国形象等。与此同时,一部 分非洲学者受到西方学界“新殖民主义”观点的影 响, 对中国在非洲的政治影响提出了批评。如 Aidoo(2012)、Mukanga(2012)等认为中国在非洲的努力 是牺牲非洲人民的利益, 与腐败的非洲国家政府做 交易, 以此达到掠夺资源的目的, 并对此提出了强 烈的批评。在探讨中非合作是否是平等对话的南 南合作模式的同时,也有部分非洲学者从非洲自身 发展角度出发, 根据中非合作探究非洲未来的发展 模式。如尼日利亚学者Obiorah(2006)从历史的角 度阐述中非合作传统, 肯定了中国对非洲国家的帮 助, 更为关键的是, 其认识到非洲应通过革新落后 的管理体系和腐败体制, 借力加快自身发展, 摆脱 资源发展的诅咒。南非学者 Prah(2007)指出每个经 济体均是平等的,提议勿用政治利益或者商业目的 评价中国在非洲的经济投资援助, 非洲应当寻找双 方合作的利益最大化等。

\section{2 从地理学角度展开的中非合作研究}

综上所述, 无论是国内, 还是国外的中非合作 研究有比较明显的学科倾向, 即对经济学和政治学 分析框架的偏好。从本文的学科切人点来说, 地理 学对正在逐渐成为研究热点的中非合作议题研究 虽然有所关注, 但仍然比较薄弱。但通过对学者学 科背景以及研究方法等研究细节的进一步梳理发 现, 地理学的分析框架, 尤其是其空间分析方法已 经逐渐被应用于中非合作相关的研究议题。

对于地理学者来说, 对中非合作及其相关议题 的关注主要基于经济地理学的分析视角,而人文地 理学其它分支学科的相关研究则少之又少。总体 来说, 地理学者们大都采用了空间分析作为其主要 方法, 而经济仍然是其主要关注的现象。例如, 尹 海伟等(2005)在对大量中国对非贸易数据资料的统 计、整理的基础上,探讨了中国对非贸易在时间和空 间序列上的变动特点, 并对中非贸易的前景进行了 
展望。分析发现, 中国对非贸易区域空间分布不平 衡, 但逐步向均衡方向发展, 贸易市场也正在逐步 多元化。采用类似的方法, 徐建刚等(2006)以 19922000 年非洲经济发展统计数据为基础, 勾勒出非洲 经济空间格局的基本特征。此外,杨文倩等(2013) 采用统计分析和空间集中度指标计算等方法, 讨论 了1992-2010年中非农产品进出口的空间特征和变 化趋势; 蒋大亮等运用地理集中度、耦合指数与区 域重心等方法分析了非洲大陆 52 个国家的空间发 展特征, 建立了区域经济社会综合发展水平指标体 系, 并对非洲人口集中指标和经济发展指标进行了 耦合计算, 在此基础上, 提出了针对非洲区域经济 社会发展的特点开展对非援助与合作的建议(蒋大 亮, 任则沛等, 2015; 蒋大亮, 杨钿等, 2015; 蒋大亮 等, 2016)。对于非洲投资环境的空间解释也逐渐 成为经济地理学的新兴研究话题, 如黎明等(2017) 利用2003-2014年中国对外直接投资(OFDI)的数据 建构面板, 通过 Tobit模型并结合实际案例,分析讨 论了中国对非直接投资的时空演化及其影响因素; 而谢守红等(2017)则从人口与就业等 42 个指标构 建了非洲国家投资环境综合评价指标体系,运用熵 权法对非洲国家的投资环境进行定量评价, 最后采 用空间聚类分析讨论了非洲投资环境的空间特 征。除采用定量研究方法外, 部分学者还利用现有 的网络数据源和跟踪访谈分析中国直接投资在多 个非洲国家所产生的影响 (Pannell, 2008; Veeck, 2008; Veeck et al, 2012)。这些来自地理学者的分析 (尤其是其对空间分析视角的强调) 无疑为中非合作 的解读提供了独特的视角。

除经济地理分析框架之外, 国内外地理学者也 有从其他人文地理学分支研究方向(如政治地理、 旅游地理等)对中非合作议题展开分析和讨论。其 中, 政治地理分析视角是一个值得关注的方向。从 国内学者来看,周晓波等(2011)讨论了中非合作过 程中石油合作的战略意义以及其对国际能源格局 的影响; 王锁劳(2006)分析了埃及的独特地理位置, 指出埃及在阿拉伯、伊斯兰和非洲等犬牙交错地区 的特殊区位注定了其应在国际政治和经济事务中 发挥独特的作用, 例如在政治合作中带头, 在经济 合作方面示范以及在文化交流方面架起桥梁等。 国外学者对中非合作的政治地理话题也有所讨论, 例如 Mawdsley (2008) 以英国报纸为例, 从批判地 缘政治的角度分析了西方媒体对 “中非合作” 以及
“西方与非洲经贸往来”的地缘政治话语建构, 反映 出西方大众媒体对中非合作的片面评论及狭险的 地缘想象; Carmody 等(2010)用“Flexigemony”(“弹 性霸权”)一词来总结和形容中国在非的能源外交 政策, 其区别于以往传统的西方霸权, 更多是流动、 多变及复杂的软性霸权, 表明这种方式更为隐秘和 更易被非洲国家接受。此外,还有少数地理学者从 旅游地理等其它人文地理学研究方向对中非合作 实践进行了探究,如尹国蔚(2013)通过图表分析和 密度计算方法, 在非洲全域、国家和地区等三个地 理尺度上, 以世界文化和自然遗产为主要旅游资源 类型, 分析了非洲世界遗产的空间分布特征; 骆高 远等(2009)从非洲旅游资源、旅游产品以及旅游市 场的现状和特征、优势和不足出发, 针对已有资源、 产品和市场的不足, 提出未来中非旅游合作的思路 和对策; Giese等(2015)从女性主义地理视角探究中 国因素如何在日常生活中影响加纳城镇女性管理 者的空间感变化。

上述文献分析说明,虽然已有的地理学研究对 中非合作话题有所关注, 但整体来说关注度仍不 够。从跨学科的角度来说, 地理学独特的空间分析 视角恰恰又能极大地补充经济学和政治学以及其 它学科对相关议题分析框架的不足。然而, 值得注 意的是,地理学对中非合作的关注似乎又没有完全 脱离经济学和政治学的学科分析框架,大量的地理 学者巧妙地将地理学的空间分析方法与经济学和 政治学的分析框架相结合,运用于经济地理学和政 治地理学对中非合作的解释。当然,这在一定程度 上也使地理学的分析框架以及人们对中非合作的 认识受到局限。

\section{4 基于人文地理学视野的中非合作研 究框架}

人文地理学作为一门研究地球表层各种人文 现象与人类活动发生过程、动态演化特征及其地域 分异规律的学科, 其核心在于探讨人地系统, 主要 包括经济地理学、文化地理学、旅游地理学、政治地 理学、社会地理学、城市地理学等分支(方创琳等, 2011)。相较于其他学科, 人文地理学更加注重分析 的综合性。通过人文地理视角对中非合作现象进 行解释,可以更全面和综合地分析该现象。如上所 述, 现有的关于中非合作的地理学文献尚未建立较 
为完整的研究框架和体系, 除经济地理学对中非合 作研究议题的关注稍系统化之外, 其他人文地理分 支的研究还比较零散。基于此, 本文将尝试从人文 地理学视角讨论与中非合作有关的潜在议题的研 究框架。由于人文地理学分支诸多, 本文将重点阐 述与中非合作实践联系最为紧密的政治地理、旅游 地理, 以及社会文化地理等三个分支。

\section{1 中非合作涉及的潜在政治地理研究议题}

国内外对政治地理较为共识的定义是“一门对 政治和地理环境之间复杂而多样的关系进行探讨 的学问”(Agnew, 1996; 安宁等, 2016)。政治地理既 讨论政治权力与不同尺度上的景观(生产或变化)之 间的关系, 谓之地方与政治议题, 又讨论地理环境 对政治活动的影响力, 谓之地缘政治。虽然有少数 研究从政治地理视角对中非合作现象进行审视(王 锁劳, 2006; Mawdsley, 2008; Carmody et al, 2010; 周晓波等, 2011), 然而这样的研究却相对比较零散, 尚未按照严格的体系化建立政治地理分析框架(如 地方与政治, 或地缘政治)。因此, 本文建议从以下 两个政治地理研究视角对中非合作进行研究:

(1) 可从地方与政治的视角对中非合作进行检 视。不同的权力来源、权力结构对于不同尺度、不 同地方的地理景观的形成或改变有不容忽视的影 响。例如, 不同非洲国家对中非合作的接受程度及 其政策反应对其所在国家、区域、城市和社区层面 的人文地理景观是否有影响? 非洲国家或其它权 力集团如何与中资企业发生互动? 反之, 中国对非 洲国家的政策是促进还是抑制了非洲人民的跨境 流动现象? 确切地, 中非合作中中国政策层面的变 化是否对中国不同地理尺度的景观产生了影响? 这些问题的答案背后的政治地理机制如何解释 等。这些议题有助于从独特的人文地理学视角理 解中非合作现象。

(2) 可从地缘政治的视角对中非合作进行研 究。作为 20 世纪最典型的第三世界区域, 加之其深 厚的殖民主义历史与文化, 非洲的政治经济形势很 大程度上反映了世界地缘政治和地缘经济格局的 变化, 牵动着大国角逐的神经。借助 “前殖民地” 的 地缘优势,英、法、美等国家长期以来在与非洲的政 治经济交流中占据着主动和主导地位, 对非洲政治 经济产生了深远影响。然而,随着中非合作的日渐 深人 (尤其是一系列中非论坛的成功举办), 中国俨 然成为一匹 “黑马”, 在对非政治经济合作交流中取
得了显著成绩。不可否认的是, 中国在非洲所倡导 的 “南南合作”, 与英、法、美等国家在非洲所倡导的 “南北合作”有很大的不同。中非合作的兴起因此 对原有的地缘政治和地缘经济格局造成了很大程 度的冲击。从这个角度来讲, 未来与中非合作议题 相关的地缘政治研究的切人点为: 中非合作的兴起 对非洲地缘政治和地缘经济格局带来了什么样的 影响? 其辩证关系是什么? 针对当下情况, 中国、 非洲和西方国家在非洲这片土地上未来的出路是 什么? 如何对非洲各个地理尺度上的政治主体及 其行为进行综合分析, 制定不同的地缘策略, 这将 是未来地缘政治细化研究的重点。

\section{2 中非合作涉及的潜在旅游地理研究议题}

旅游地理是地理学和旅游学之间的交叉学科, 既研究人的休闲活动, 又讨论其与地理环境之间的 关系, 对旅游产生的条件和地理背景、旅游资源评 价、旅游区划和规划、旅游产业发展、旅游者空间行 为、旅游营销、旅游影响、旅游与地方 (国家、地区、 城市和社区)的发展等, 是一门极具综合和应用性 的方向(黄震方等, 2015)。随着现代社会人们物质 生活水平日益提高, 旅游逐渐成为人们生活不可或 缺的一部分。随着中非合作的深人,中国和非洲国 家之间的经济贸易关系越来越紧密, 随之而来的服 务产业也愈加繁荣。以旅游行业为例, 越来越多的 非洲人将中国视为理想的旅游目的地, 同时也有越 来越多的中国人将非洲视为神秘的旅游目的地。 伴随着这样的现象, 与中非合作相关的旅游地理研 究话题也日渐浮现。然而, 既有研究很少从旅游地 理的角度对中非合作进行讨论。借鉴旅游地理相 关框架, 本文认为中非合作议题可从下述几个方面 展开:

(1) 把握非洲地区旅游资源及其(人文)地理环 境的特征, 建立相关旅游资源和旅游市场的评价体 系,在此基础上将非洲旅游系统的复杂特征应用于 旅游开发、经营和管理中。一些具体的问题如: 非 洲分地区旅游资源特征是什么? 分别适合于什么 样的旅游产品设计? 这些旅游资源的人文地理环 境是否适合做相关的旅游产品设计? 非洲分地区 旅游开发、经营和管理过程中可能面临的问题与挑 战? 这些问题的研究可在智力和应用层面为中非 合作实践作出一定贡献。

(2) 从旅游者空间行为以及旅游体验等角度出 发, 探讨中非合作过程中旅游产业发展的可能性。 
不论是针对中国还是全球旅游市场来说, 非洲作为 旅游目的地似乎都很难绕开其刻板的印象。然而, 近年来, 对于中国游客来说, 一部分非洲国家正在 成为新的旅游热点。中国游客对非旅游动机、旅游 动力、旅游决策、旅游偏好变化背后的因素和机制 是什么? 反过来, 在中国的各个旅游目的地也经常 看到越来越多来自非洲的游客, 中国旅游非洲市场 规模日渐增长的背后, 非洲旅游者行为动机、动力、 决策和偏好又是什么? 这些问题的探讨有助于从 旅游者的视角出发进一步了解旅游者, 了解旅游产 品和市场, 积极调动人的休闲和旅游需求, 充分发 现和利用既有的旅游资源, 助力于中非合作空间实 践的进一步完善和发展。

\section{3 中非合作涉及的潜在社会文化地理研究议题}

社会地理强调从地理学的视角对人类社会现 象、特征、社会集团分布和差异进行分析, 而文化地 理则关注人类文化的空间组合, 讨论人类文化与地 理环境(地方和区域)之间的相互关系 (Pain et al, 2001; Anderson et al, 2003)。当今, 社会与文化地理 学研究议题往往集中于社会契约、社会生产、社会 身份、社会公平、空间正义、空间的意义和象征、景 观生产、跨境流动、移民、种族、种群差异、文化表征 以及文化政治等话题(Pain et al, 2001; Anderson et al, 2003)。中非两地文化差异大, 涉及的民族、种 族、族群和宗教众多, 而中非合作带来了两地之间 文化和社会的激烈交流与碰撞, 势必产生许多新的 社会与文化地理研究话题。现有的中非合作研究 往往将关注的重心侧重于政治和经济层面, 而文化 则视为经贸与政治合作的附属品被忽视。然而, 社 会与文化的交流在很大程度上可以促进中非两地 经贸的发展。因此, 本文建议可从社会与文化地理 视角对中非合作展开分析：

(1) 针对部分西方学者和非洲学者对中国所提 出的 “南南合作” 模式的挑战, 本文认为可从社会地 理学的角度, 从微观视角对中非合作及其在细节层 面的社会影响进行讨论, 对其“新殖民主义”等歪曲 中非合作的言论作出正面有力的回击。总体来讲, 中非合作给非洲地区不同尺度上的社会群体带来 了很多积极和正面的影响, 这与传统发达国家势力 在非的情况完全不同。此外, 中非合作间中非双边 移民管理、移民的身份认同以及移民与迁人地的地 方协商等议题也是伴随着中非合作日渐成熟而带 来的社会地理话题, 例如, 在华非洲移民、在非中国
移民与当地居民间的协商与自我身份认同情况, 非 洲本地居民和中国员工等行为主体对在非中资企 业形成的飞地现象的空间感知等。

(2) 中非文化交流有助于推动中非民众对双方 文化的认同与理解,进一步助推其他中非合作内容 的发展。因此, 从文化地理视角可从以下几方面对 中非合作进行讨论, 如: 中非合作带来的文化差异 性、文化融合问题以及文化多元主义的分析, 具体 如中国移民和资本的大量涌人对非洲当地文化带 来的空间变化等; 中国、西方、非洲等不同媒体对中 非合作状况的表述, 以及关于中非合作的话语分 析。例如, 在非中国移民听到多种媒体的声音, 结 合自身体会得出不一样的国家认同和地方认同感 等; 此外,中非移民的文化风俗、饮食习惯等差异和 融合对双方交流带来的影响和景观塑造也是有意 义的文化地理课题等。

最后, 值得强调的是, 上述话题之间并没有明 确的界限, 而且, 人文地理视角的研究并不限于上 述话题。上文仅仅从一些与中非合作密切相关、同 时又是人文地理学重要分支的视角对中非合作潜 在的研究话题提出一些建议, 以期从地理学的视角 对进一步推进中非合作作出应有的贡献。

\section{5 结论}

本文通过文献梳理的方法讨论了目前国内外 关于中非合作研究的文献情况。整体来看,关于中 非合作议题的研究已受到国内外学者的广泛关注, 且呈现增长趋势。具体来说, 中西非学者都从自身 不同的角度出发, 对中非合作现象进行了讨论。这 些文献涉及中非合作具体的经济增长模式、援助情 况、社会经济效应以及环境效应等,也关注了中非 合作事件及其对非洲、西方国家和中国多方参与的 地缘政治和地缘经济格局的影响。然而,现有的文 献大部分是基于政治、经济学研究框架对中非合作 现象的讨论, 包括少量的地理学研究文献亦如此, 其研究的视角仍有待扩展。正因如此, 地理学独特 的空间分析方法可为理解中非合作现象提供不同 于经济学和政治学的视角, 可在很大程度上丰富已 有的中非合作研究。此外, 从知识生产的地理学角 度来分析, 发现尽管部分中国和非洲学者从“南南 合作”视角对中非合作进行了解读, 但关于中非合 作的研究(尤其在国际话语权的层面)仍存在“流讯 
援助” “新殖民主义”以及“新资本主义”等学术方面 的话语霸权, 并影响到非洲学者群体。从这个层面 来看, 中国学者更需从多维度、多学科层面(尤其是 在国际学术话语层面) 争取更多的学术话语权, 通 过学理的梳理积极回应西方的学术话语霸权及其 影响, 以此为国家发展作出贡献。

在国际政治和经济舞台上,中非合作将成为世 界发展的一个焦点。本文从“中非合作”研究的必 要性、“中非合作”已有的研究概述、“中非合作”可 能涉及的人文地理学研究话题等方面对中非合作 进行分析, 并从政治地理、旅游地理, 及社会和文化 地理等角度探讨可能成为未来中非合作研究的热 点话题。与西方学者、甚至非洲学者相比, 虽然中 国学者对中非合作研究较早, 但缺乏系统性和理论 性的研究体系。随着中国在多个大学成立非洲研 究中心, 希望未来来自中国学者的声音, 尤其是人 文地理学者的声音能越来越强。

中非合作作为现今中国倡议的南南合作典范, 如何使其进一步深化并发展得越来越好, 是自古以 来以经世致用为目标的人文地理学者的责任。希 望未来能有越来越多的人文地理学者投身于中非 合作研究, 为中国的中非合作研究理论化、体系化 作出自己的贡献。与此同时, 本文所提出和讨论的 中非合作地理学分析框架在很大程度上呼应了地 理学“走出国门”的大国智力责任和担当。

\section{参考文献(References)}

安宁, 钱俊希, 陈晓亮, 等. 2016. 国际上的政治地理学研究 进展与启示: 对《Political Geography》杂志 2005-2015 年 载文的分析 [J]. 地理学报, 71(2): 217-235. [An N, Qian J X, Chen X L, et al. 2016. Progress in recent international political geography research: Towards an analysis of journal papers in Political Geography[J]. Acta Geographica Sinica, 71(2): 217-235.]

杜德斌, 马亚华. 2015. “一带一路”: 中华民族复兴的地缘大 战略 [J]. 地理研究, 34(6): 1005-1014. [Du D B, Ma Y H. 2015. "One Belt and One Road": The grand geo-strategy of China's rise[J]. Geographical Research, 34(6): 10051014.]

方创琳, 周尚意, 柴彦威, 等. 2011. 中国人文地理学研究进 展与展望[J]. 地理科学进展, 30(12): 1470-1478. [Fan C L, Zhou S Y, Chai Y W, et al. 2011. Updated progress and perspectives of the discipline of human geography in China [J]. Progress in Geography, 30(12): 1470-1478. ]

傅政罗. 1996. 发展中非经贸合作的新机遇 [J]. 国际贸易,
(11): 31-32. [Fu Z L. 1996. Fazhan Zhongfei jingmao hezuo de xinjiyu[J]. Intertrade, (11): 31-32.]

贺鉴, 段钰琳. 2017. 论中非海洋渔业合作 $[\mathrm{J}]$. 中国海洋大学 学报: 社会科学版, (1): 36-42. [He J, Duan Y L. 2017. On the fishery cooperation between China and Africa[J]. Journal of Ocean University of China: Social Science, (1): 3642.]

胡美, 刘鸿武. 2012. 中国援非五十年与中国南南合作理念 的成长 $[\mathrm{J}]$. 国际问题研究, (1): 12-28. [Hu M, Liu H W. 2012. Zhongguo yuanfei 50 nian yu Zhongguo Nannan hezuo linian de chengzhang[J]. International Studies, (1): 1228.]

黄梅波, 唐露萍. 2013. 南南合作与南北援助: 动机、模式与 效果比较[J]. 国际展望, (3): 8-26, 135. [Huang M B, Tang L P. 2013. On South-South cooperation and North-South cooperation in international aid architecture[J]. International Review, (3): 8-26, 135.]

黄梅波, 唐正明. 2016. 中非产能合作的金融需求及中非金 融合作的推进[J]. 国际经济评论, (4): 91-107. [Huang M B, Tang Z M. 2016. Financial needs of China-Africa capacity cooperation and promotion of the China- Africa financial cooperation[J]. International Economic Review, (4): 91-107.]

黄泽全. 1992a. 中非经济合作与文化交流[J]. 西亚非洲, (3): 73-76, 40. [Huang Z Q. 1992a. Zhongfei jingji hezuo yu wenhua jiaoliu[J]. West Asia and Africa, (3): 73-76, 40.]

黄泽全. 1992b. 中非友好合作四十二年 [J]. 西亚非洲, (1): 58-62, 66. [Huang Z Q. 1992b. Zhongfei youhao hezuo 42 nian[J]. West Asia and Africa, (1): 58-62, 66.]

黄震方, 黄員. 2015. 基于人地关系的旅游地理学理论透视 与学术创新 [J]. 地理研究, 34(1): 15-26. [Huang Z F, Huang R. 2015. The theoretical perspective and academic innovation of tourism geography based on human-environment interactions[J]. Geographical Research, 34(1): 15-26.] 蒋大亮, 任航, 张凌华, 等. 2016. 非洲地区人口与经济空间 耦合研究 [J]. 热带地理, 36(5): 823-832. [Jiang D L, Ren H, Zhang L H, et al. 2016. Coupling relationship between population and economy in Africa[J]. Tropical Geography, 36(5): 823-832.]

蒋大亮, 任则沛, 张振克. 2015. 非洲区域经济发展差异时空 变化研究 [J]. 世界地理研究, 24(3): 34-41. [Jiang D L, Ren Z P, Zhang Z K. 2015. Study on the spatio-temporal change of regional economic development in Africa[J]. World Regional Studies, 24(3): 34-41.]

蒋大亮, 杨钿, 任则沛, 等. 2015. 基于 TOPSIS 法的非洲区域 经济社会综合发展水平评价 [J]. 热带地理, 35(2): 242249. [Jiang D L, Yang D, Ren Z P. 2015. Evaluation on the 
comprehensive economic and social development of Africa with the method TOPSIS[J]. Tropical Geography, 35(2): 242-249.]

黎明, 夏昕鸣, 朱晟君, 等. 2017. 中国对非直接投资时空演 化及其影响因素 [J]. 经济地理, 37(11): 19-27. [Li M, Xia X M, Zhu S J, et al. 2017. The temporal and spatial evolution of China's foreign direct investment in Africa and its influencing factors[J]. Economic Geography, 37(11): 1927.]

李安山. 2015. 中非合作的基础: 民间交往的历史、成就与特 点 [J]. 西亚非洲, (3): 51-73. [Li A S. 2015. The foundation of China-Africa cooperation: History, achievement and feature of non-governmental exchanges[J]. West Asia and Africa, (3): 51-73.]

刘鸿武, 卢凌宇. 2013. “中国梦”与 “非洲梦”: 中非命运共同 体的建构 [J]. 西亚非洲, (6): 19-33. [Liu H W, Lu L Y. 2013. "Chinese Dream" and "African Dream": The construction of China-Africa Community of Destiny[J]. West Asia and Africa, (6): 19-33.]

陆瑾. 2015. 历史与现实视國下的中伊合作: 基于伊朗人对 “一带一路”认知的解读 $[\mathrm{J}]$. 西亚非洲, (6): 53-69. [Lu J. 2015. China Iranian cooperation in history and modern times: An explanation based on Iranians views of "The Belt and Road" Initiative[J]. West Asia and Africa, (6): 53-69.] 罗建波. 2009. 中国与西方国家的对非洲外交: 在分歧中寻 求共识与合作 $[\mathrm{J}]$. 世界经济与政治, 23(4): 26-35. [Luo J B. 2009. China \& Western countries' diplomacy with Africa: Seeking consensus and cooperation from differences [J]. World Economics and Politics, 23(4): 26-35.]

罗建波. 2013. 中非关系与中国的世界责任 [J]. 世界经济与 政治, (9): 52-70. [Luo J B. 2013. China-Africa relations and China's global responsibility[J]. World Economics and Politics, (9): 52-70.]

骆高远, 陆林. 2009. 中非旅游合作的现状和未来 [J]. 地理科 学, 29(2): 200-205. [Luo G Y, Lu L. 2009. Current situation and future of China-Africa tour cooperation[J]. Scientia Geographica Sinica, 29(2): 200-205.]

孟辽阔. 2015. “一带一路”视野下的巴基斯坦战略地位及其 实现路径探析 [J]. 世界经济与政治论坛, (4): 29-45. [Meng L K. 2015. "Yidai Yilu" shiyexia de Bajisitan zhanlue diwei jiqi shixian lujing tanxi[J]. Forum of World Economics \& Politics, (4): 29-45.]

牛长松. 2014. 孔子学院与中国对非语言文化外交[J]. 西亚 非洲, (1): 64-78. [Niu C S. 2014. Confucius Institutes and China's lingustical and cultural diplomacy to Africa[J]. West Asia and Africa, (1): 64-78.]

汪勤梅. 1998. 中非经贸合作飞跃发展的 20 年 [J]. 西亚非洲,
(5): 53-54. [Wang Q M. 1998. Zhongfei jingmao hezuo feiyue fazhan de 20nian[J]. West Asia and Africa, (5): 53-54.] 汪文卿, 赵忠秀. 2014. 中非合作对撒哈拉以南非洲国家经 济增长的影响: 贸易、直接投资与援助作用的实证分析 [J]. 国际贸易问题, (12): 68-79. [Wang W Q, Zhao Z X. 2014. The impact of China-Africa cooperation on economic growth of Sub-Saharan Africa countries- the role of trade, FDI and aid[J]. Journal of International Trade, (12): 68-79.]

王洪一. 2006. 试论“中国威胁论” [J]. 西亚非洲, (8): 28-32. [Wang H Y. 2006. Shilun “Zhongguo weixielun” [J]. West Asia and Africa, (8): 28-32.]

王南. 2015. 非洲: “一带一路”不可或缺的参与者 [J]. 亚太安 全与海洋研究, (5): 97-109, 128. [Wang N. 2015. Africa: The indispensable participant for "One Belt and One Road" [J]. Asia- Pacific Security and Maritime Affairs, (5): 97109, 128.]

王锁劳. 2006. 埃及的独特地位及其在中非合作中的有效作 用[J]. 国际政治研究, 43(4): 80-94. [Wang S L. 2006. Aiji de dute diwei jiqi zai Zhongfeihezuo zhong youxiao zuoyong[J]. International Political Studies, 43(4): 80-94.]

谢守红, 甘晨, 王庆. 2017. 非洲国家投资环境综合评价及空 间差异分析 [J]. 经济地理, 37(8): 10-16. [Xie S H, Gan C, Wang Q. 2017. Comprehensive evaluation of investment environment in African countries and spatial difference analysis[J]. Economic Geography, 37(8): 10-16.]

徐建刚, 尹海伟, 钟桂芬, 等. 2006. 基于空间自相关的非洲 经济格局 $[\mathrm{J}]$. 经济地理, 26(5): 771-775. [Xu J G, Yin H W, Zhong G F, et al. 2006. Study on African economy structure based on spatial autocorrelation[J]. Economic Geography, 26(5): 771-775.]

央视网. 2016. 中非合作硕果累累[N/OL]. 2016-07-30 [2018-

04- 01]. http://tv.cntv.cn/video/VSET100283759803/. [CCTV.com. 2016. Zhongfei hezuo shuoguoleilei[N/OL]. 2016- 07- 30 [2018- 04- 01]. http://tv.cntv.cn/video/ VSET100283759803/.]

杨文倩, 杨军, 王晓兵. 2013. 中非农产品贸易国别变化时空 分析 [J]. 地理研究, 32(7): 1316-1324. [Yang W Q, Yang J, Wang X B. 2013. The temporal-spatial distribution of agricultural trade between China and Africa[J]. Geographical Research, 32(7): 1316-1324.]

姚桂梅. 2008. 中非合作论坛及其经贸影响 [J]. 亚非纵横, (1): 24-29. [Yao G M. 2008. Forum on China-Africa cooperation and its impact on bilateral economic and trade relations[J]. Asia \& Africa Review, (1): 24-29.]

姚桂梅. 2013. 中国对非洲投资合作的主要模式及挑战 $[\mathrm{J}]$. 西亚非洲, (5): 103-117. [Yao G M. 2013. Main models 
and challenges of Sino- Africa investment cooperation[J]. West Asia and Africa, (5): 103-117.]

尹国蔚. 2013. 非洲世界遗产的分布特征 [J]. 地理科学进展, 32(6): 958-966. [Yin G W. 2013. Geographical distribution characteristics of African world heritages[J]. Progress in Geography, 32(6): 958-966.]

尹海伟, 徐建刚, 曾尊固, 等. 2005. 中国对非贸易时空分异 研究 [J]. 经济地理, 25(1): 44-48. [Yin H W, Xu J G, Zeng Z G, et al. 2005. Study on space-time difference of SinoAfrican trade[J]. Economic Geography, 25(1): 44-48.]

张菲. 2013. 中非经贸合作区建设模式与可持续发展问题研 究 [J]. 国际贸易, (3): 34-39. [Zhang F. 2013. The constructing model of China-Africa trade and the research on its sustainability[J]. Intertrade, (3): 34-39.]

张海冰. 2006. 中非合作与南南合作 $[\mathrm{J}]$. 毛泽东邓小平理论 研究, (12): 65-68. [Zhang H B. 2006. China-Africa cooperation and South-South cooperation[J]. Studies on Maoist and Deng Xiaoping Theories, (12): 65-68.]

张忠祥. 2009. 中非合作论坛框架下的农业合作 [J]. 国际展 望, (2): 103-112. [Zhang Z X. 2009. Sino-Africa agriculture cooperation under FOCAC framework[J]. Global Review, (2): 103-112.]

周文, 赵方. 2017. 中国"一带一路"倡议下的中非合作是"新 殖民主义"吗? [J]. 马克思主义研究, (1): 129-142, 152. [Zhou W, Zhao F. 2017. Zhongguo "Yidai Yilu" changyi xia de Zhongfei hezuo shi "Xin Zhimin zhuyi" ma?[J]. Research on Marxism, (1): 129-142,152.]

周晓波, 吴家新, 赵媛. 2011. 中非石油合作的战略意义与重 点区域选择 [J]. 世界地理研究, 20(1): 63-69. [Zhou X B, Wu J X, Zhao Y. 2011. The strategic significance of ChinaAfrica oil cooperation and key areas selection[J]. World Regional Studies, 20(1): 63-69.]

Aidoo R. 2012. China's image problem in Africa[N]. The Diplomat, 2012-10-25.

Agnew J. 1996. Political geography: A reader[M]. London, UK: Edward Arnold.

Amadi L. 2012. Africa, beyond the 'new' dependency: A political economy[J]. African Journal of Political Science and International Relations, 6(8): 191-203.

Anderson K, Domosh M, Pile S, et al. 2003. Handbook of cultural geography[M]. London, UK: Sage.

Asche H. 2008. Contours of China's "Africa Mode" and who may benefit[J]. Journal of Current Chinese Affairs, (3): 165-180.

Berger B. 2006. China's engagement in Africa: Can the EU sit back?[J]. South African Journal of International Affairs, 13
(1): $115-127$.

Campbell H. 2008. China in Africa: Challenging US global hegemony[J]. Third World Quarterly, 29(1): 89-105.

Carmody P R, Owusu F. 2007. Competing hegemons? Chinese versus American geo-economic strategies in Africa[J]. Political Geography, 26(5): 504-524.

Carmody P R, Taylor I. 2010. Flexigemony and force in China's resource diplomacy in Africa: Sudan and Zambia compared[J]. Geopolitics, 15(3): 496-515.

Giese K, Thiel A. 2015. Chinese factor in the space, place and agency of female head porters in urban Chana[J]. Social \& Cultural Geography, 16(4): 444-464.

Grimm S, Hackenesch C. 2017. China in Africa: What challenges for a reforming European Union development policy? Illustrations from country cases[J]. Development Policy Review, 35(4): 549-566.

Haglund D. 2009. In it for the long term? Governance and learning among Chinese investors in Zambia's copper sector[J]. China Quarterly, 199(199): 627-646.

Jaff A M, Lewis S W. 2002. Beijing's oil diplomacy[J]. Survival, 44(1): 115-133.

Jauch H. 2011. Chinese investments in Africa: Twenty- first century colonialism?[J]. New Labor Forum, 20(2): 48-55.

Kärkkäinen A. 2016. Does China have a geo-economic strategy towards Zimbabwe? The case of the Zimbabwean natural resource sector[J]. Asia Europe Journal, 14(2): 185-202.

Larkin B D. 1973. China and Africa 1949-1970: The foreign policy of the People's Republic of China[M]. Berkeley, CA: University of California Press.

Lee C K. 2009. Raw encounters: Chinese managers, African workers and the politics of casualization in Africa's Chinese enclaves[J]. The China Quarterly, (199): 647-666.

Manji F M, Marks S. 2007. African perspectives on China in Africa[M]. Oxford, UK: Fahamu.

Mawdsley E. 2008. Fu Manchu versus Dr Livingstone in the dark continent? Representing China, Africa and the West in British broadsheet newspapers[J]. Political Geography, 27(5): 509-529.

Meidan M. 2006. China's Africa policy: Business now, politics later[J]. Asian Perspective, (30): 69-93.

Montinari L, Prodi G. 2011.China's impact on Intra- African trade[J]. Chinese Economy, 44(4): 75-91.

Mukanga C. 2012. China's new colonialism in Africa[N/OL]. Zambian Economist. 2012-05-17[2018-04-01]. http://www. zambian- economist.com/2012/05/chinas- new- colonialismin-africa.html.

Mung E M. 2008. Chinese migration and China's foreign poli- 
cy in China[J]. Journal of Chinese Overseas, 4(1): 91-109. Naim M. 2007. Rogue aid[J]. Foreign Policy, (159): 95-96.

Obiorah N. 2006. Who's afraid of China in Africa?[N/OL]. Pambazuka News, 2006-12-14[2018-04-01]. https://www. pambazuka.org/food-health/who's-afraid-china-africa.

Osci-Hwede B Z. 2012. The dynamics of China-Africa cooperation[J]. Afro Asian Journal of Social Sciences, 3(1): 1-25.

Pain R, Barke M, Gough J, et al. 2001. Introducing social geographies[M]. London, UK: Arnold.

Pannell C W. 2008. China's economic and political penetration in Africa[J]. Eurasian Geography and Economics, 49(6): 706-730.

Prah K K. 2007. China and Africa: Defining a relationship[J].
Development, 50(3): 69-75.

Sautman B, Yan H. 2007. Friends and interests: China's distinctive links with Africa[J]. African Studies Review, 50(3): 75-114.

Tan-Mullins M, Mohan G, Power M. 2010. Redefining 'aid' in the China- Africa context[J]. Development \& Change, 41 (5): $857-881$.

Veeck G. 2008. China's exports and imports of agricultural products under the WTO[J]. Eurasian Geography and Economics, 49(5): 569-585.

Veeck G, Diop S H A. 2012. Chinese engagement with Africa: The case of Madagascar[J]. Eurasian Geography and Economics, 53(3): 400-418.

\title{
Chinese geography "goes out": Looking at China-Africa cooperation from the perspective of human geography
}

\author{
AN Ning ${ }^{1}$, LIANG Bangxing ${ }^{2}$, ZHU Hong ${ }^{1 *}$ \\ (1. Centre for Human Geography and Urban Development, School of Geographical Sciences, Guangzhou \\ University, Guangzhou 510006, China; 2. School of Geography, South China Normal University, Guangzhou \\ 510631, China)
}

\begin{abstract}
China- Africa cooperation is becoming an unavoidable international political and economic event, creating a large number of research topics. This study adopted the method of Endnote analysis to examine the relevant literature from four well-known international and Chinese academic publication databases. Based on a fuzzy keyword search with the key words of China-Africa cooperation, Sino-Africa cooperation, China-Africa collaboration, and Sino- Africa collaboration in Web of Science, Taylor \& Francis, and Elsevier and zhongfei hezuo in CNKI, this study collected 241 and 382 publications from the international and Chinese databases, respectively. The two main conclusions are as follows: (1) Both international and Chinese scholars have paid much attention to the political and economic aspects of China-Africa cooperation, situating this theme into the framework of political science and economy for discussion, while the research perspective from other subjects seem to have been underexamined; (2) Although a small number of scholars have explored China- Africa cooperation from the perspective of geography and spatial analysis, such kind of studies seem to have seldom departed from the analytical framework of political science and economics. Human geography studies are also small in number and unsystematic. In this regard, this article proposes potential future research directions to discuss this topic, in particular from the lens of political geography, tourism geography, and social and cultural geography, which may gradually become hotspot topics in the near future. This article advocates for increased human geographer participation in the research of China-Africa cooperation thereby contributing to the study and practice of this cooperation. It also encourages human geographers in China to respond to China's growing intellectual responsibility as a great power "going out."
\end{abstract}

Key words: China-Africa cooperation; human geography; reference analysis; going out 